\title{
Correlation and Relativistic Effects on the Level Structure of Negative Ions of Atoms with Half-filled p Shell
}

\author{
Gülay Günday Konan*, Leyla Özdemir \& Nurgül Gündüz Atik \\ Physics Department, Faculty of Science and Art, \\ Sakarya University, 54187, Sakarya, Turkey \\ Email: ggunday@sakarya.edu.tr
}

\begin{abstract}
The level structure for negative ions (anions) of some atoms with halffilled $\mathrm{p}$ shell have been investigated using the multiconfiguration Hartree-Fock method with Breit-Pauli relativistic effects ( $\mathrm{MCHF}+\mathrm{BP}$ method). Thus, we have performed a systematic calculation including correlation and relativistic corrections for negative ions of these atomic systems. Investigations into the level structures of negative ions provide valuable insight into the fundamental problem of many-body motion. The correlation and relativistic effects in negative ions relative to neutral atoms and positive ions are greatly expected. In this work, we also discuss calculations for these effects in view of the $\mathrm{MCHF}+\mathrm{BP}$ method.
\end{abstract}

Keywords: Breit-Pauli Hamiltonian; correlation and relativistic effects; electron affinity; fine-structure levels; MCHF method; negative ion.

\section{$1 \quad$ Introduction}

The spectroscopy of negative ions has been an area of considerable interest due to the developments in theoretical methods and experimental techniques. Investigations into the dynamics of negative ions (anions) provide valuable insight into the fundamental problem of many-body motion, which is critical for a detailed understanding of the electronic structure of atoms and molecules. Negative ions are fragile atomic systems that differ from neutral atoms and positive ions in many important aspects [1-3]. The total energy $E$ of an atom includes the correlation and relativistic corrections in added non-relativistic energy. Results with improved accuracy from this form have been found for neutral atoms not only in the ground state but also in low-lying excited states, as well as singly charged cations and anions [4]. The binding energy of the outermost electron is considerably smaller than in the isoelectronic atom due to the absence of the long-range Coulomb attraction in negative ions. In this case the electron correlation will be much more important, and negative ions are suitable test objects for various atomic theories [3]. The influences of correlation effects are very important in negative ions relative to neutral and positive ions.

Received June $8^{\text {th }}, 2012$, Revised February $19^{\text {th }}, 2013$, Accepted for publication March $6^{\text {th }}, 2013$.

Copyright (C) 2013 Published by ITB Journal Publisher, ISSN: 2337-5760, DOI: 10.5614/j.math.fund.sci.2013.45.2.1 
As determined in [3], the properties of most atomic negative ions, including their binding energies and electronic structures, are now well established. But, some electron affinities and fine-structure splittings are not known with complete accuracy. In contrast to the infinite number of states in atoms, the short-range potential of negative ions typically sustains only a few bound states. Fine-structure splittings of many negative ions are poorly determined.

In this paper we systematically report the correlation and relativistic corrections to electron affinities of atoms with half-filled $\mathrm{p}$ shell, treating an approximate Schrödinger form of the Breit-Pauli Hamiltonian as a perturbation [5], and investigate the fine-structure levels of the ground states of the anions of these atoms. The calculations have been carried out using the MCHF atomic structure package developed by Fischer [6]. The ground state valence configuration of neutral atoms with half-filled $p$ shell is $n p^{34} S_{3 / 2}$, and the configuration of the negative ions of these atoms is $\mathrm{np}^{43} \mathrm{P}_{2,1,0}$ with the lowest energy level being $\mathrm{J}=2$, followed by $\mathrm{J}=1$ and then $\mathrm{J}=0$. We present the electron affinities and finestructure splittings for negative ions of nitrogen and phosphorus, which have ground states $2 \mathrm{p}^{43} \mathrm{P}_{2}$ and $3 \mathrm{p}^{43} \mathrm{P}_{2}$, respectively, according to various configuration sets [7]. For this reason, here we give only the electron affinities of arsenic (As, $\mathrm{Z}=33)$, antimony $(\mathrm{Sb}, \mathrm{Z}=51)$ and bismuth $(\mathrm{Bi}, \mathrm{Z}=83)$, and the fine-structure levels of the ground state for the negative ions of these atoms $\left(\mathrm{As}^{-}, \mathrm{Sb}^{-}\right.$and $\mathrm{Bi}^{-}$, respectively). We have made the large-scale calculations according to various configuration sets for considering correlation effects. We present only one calculation result for antimony and bismuth because the extensive configuration state functions are produced from other configuration sets. In the calculation for antimony and bismuth presented here, we have considered the configuration set proposed by Biemont and Quinet [8]. The configurations used in the calculations for atoms (As, $\mathrm{Sb}$ and $\mathrm{Bi})$ and negative ions $\left(\mathrm{As}^{-}, \mathrm{Sb}^{-}\right.$and $\left.\mathrm{Bi}^{-}\right)$are given in Table 1.

The data on half-filled subshell negative ions can be found on the NIST website [9], and a review on structure, dynamics and collisions of atomic negative ions is presented by Andersen [10]. The binding energies of the ground state in $\mathrm{As}^{-}$, $4 \mathrm{p}^{43} \mathrm{P}_{2}$, and the $4 \mathrm{p}^{43} \mathrm{P}_{0}$ state have previously been determined using broadband tunable threshold spectroscopy by Feldmann, et al. [11]. This measurement yielded an electron affinity of $0.81(3) \mathrm{eV}$ and a fine-structure splitting $\Delta \mathrm{E}\left({ }^{3} \mathrm{P}_{0}\right.$ $\left.-{ }^{3} \mathrm{P}_{2}\right)=0.17 \mathrm{eV}$. Lippa, et al. [12] used photoelectron spectroscopy to measure the electron affinity of As to be $814(8) \mathrm{meV}$. Both of these studies also determined the fine structure intervals in the negative ion. The energies of the fine-structure components $4 \mathrm{p}^{43} \mathrm{P}_{1}$ and $4 \mathrm{p}^{43} \mathrm{P}_{0}$ of $\mathrm{As}^{-}$have been determined relative to the $4 \mathrm{p}^{43} \mathrm{P}_{2}$ ground state by Haeffler, et al. [3], using photodetachment electron spectroscopy. Fine-structure splittings of $\Delta \mathrm{E}\left({ }^{3} \mathrm{P}_{1}-{ }^{3} \mathrm{P}_{2}\right)=125(3) \mathrm{meV}$ and $\Delta \mathrm{E}\left({ }^{3} \mathrm{P}_{0}-{ }^{3} \mathrm{P}_{2}\right)=166(5) \mathrm{meV}$ were obtained in [3]. This was the first 
experimental determination of the energy of the $\mathrm{J}=1$ level and an improvement of the accuracy for the $\mathrm{J}=0$ level. Walter, et al. [13] measured the threshold energies for detachment of each of the three fine structure levels of As ${ }^{-}$yielding the electron affinity of As and energy separations of the levels using tunable photodetachment threshold spectroscopy. Koga, et al. [4] found a negative contribution of ionization potential for As.

The experimental study of the $\mathrm{Sb}^{-}$ion by Scheer, et al. [14] proved that a combination of one-, two-, and three-photon detachment experiments can be used to completely and accurately determine the energies of the bound terms $\left({ }^{3} \mathrm{P}\right.$ and ${ }^{1} \mathrm{D}$ ) and the fine-structure levels of the ${ }^{3} \mathrm{P}$ term. This study clearly demonstrated the potential of utilizing forbidden transitions in optical studies of negative. In [14], the hyperfine-structure parameters were also calculated using relativistic configuration interaction calculations, which yielded energy values for the ${ }^{3} \mathrm{P}_{1},{ }^{3} \mathrm{P}_{0}$, and ${ }^{1} \mathrm{D}_{2}$ levels in good agreement with the experimentally determined energy splittings as well. Feldmann, et al. [11] and Koga, et al. [4] presented the same investigations for $\mathrm{Sb}^{-}$as well as $\mathrm{As}^{-}$.

Feigerle, et al. [15] obtained the electron affinity of $\mathrm{Bi}^{-}$using photodetachment electron spectrometry. Bilodeau and Haugen reported the results of highresolution infrared laser photodetachment threshold experiments on the negative ion of bismuth [16]. The hyperfine structure of the neutral and negative-ion ground states is included in the threshold model. The electron affinity of ${ }^{209} \mathrm{Bi}$ is determined to be $7600.66(10) \mathrm{cm}^{-1}$ [942.362(13) meV]. The photodetachment cross sections of $\mathrm{Bi}$ ion were also measured by Feldmann, et al. [11].

\section{Calculation Method}

In the multiconfiguration Hartree-Fock (MCHF) approximation [5], the Hamiltonian is used for obtaining the best radial functions for the set of nonrelativistic energies of the interacting terms. The wave function is expressed as a linear combination of orthonormal configuration state functions (CSFs) so that

$$
\Psi(\gamma L S)=\sum_{i=1}^{M} c_{i} \Phi\left(\gamma_{i} L S\right), \sum_{i=1}^{M} c_{i}^{2}=1
$$

In this expansion $\Phi\left(\gamma_{i} L S\right), \gamma_{i}$, and $c_{i}$ represent configuration state function in LS coupling, configurations, and mixing coefficients of configurations, respectively. Then the non-relativistic energy expression becomes

$$
\mathcal{E}(\gamma L S)=\sum_{i=1}^{M} \sum_{j=1}^{M} c_{i} c_{j}\left\langle\Phi\left(\gamma_{i} L S\right)|\boldsymbol{H}| \Phi\left(\gamma_{j} L S\right)\right\rangle
$$


If the interaction matrix $\mathbf{H}=\left(H_{i j}\right)$ and a column vector of the expansion coefficients (or mixing coefficients) $\mathbf{c}=\left(c_{1}, \ldots, c_{M}\right)^{t}$, the energy of system is

$$
E=\mathbf{c}^{t} \mathbf{H c} .
$$

The energy functional will depend on both $\mathbf{P}$ (the column vector of radial functions) and $\mathbf{c}$, since $H_{i j}$ depends on the radial functions. A solution of the MCHF problem simultaneously requires the solution of the secular equation and the variation radial equations. If the secular problem is solved it is called a configuration interaction (CI) calculation. If any radial function is optimized, it is called a multiconfiguration Hartree-Fock calculation. Therefore it takes into account correlation effects.

The Breit-Pauli Hamiltonian includes relativistic effects. This Hamiltonian can be written as

$$
H_{B P}=H_{N R}+H_{R S}+H_{F S},
$$

where $H_{N R}, H_{R S}$, and $H_{F S}$ are the non-relativistic many-electron Hamiltonian, the relativistic shift Hamiltonian, and fine-structure Hamiltonian, respectively. In atomic units, $H_{N R}$ is

$$
H_{N R}=\sum_{i=1}^{N}\left(-\frac{1}{2} \nabla_{i}^{2}-\frac{Z}{r_{i}}\right)+\sum_{i>j} \frac{1}{r_{i j}}
$$

$H_{R S}$ is the relativistic shift operator and including mass correction, one-and twobody Darwin terms, spin-spin contact term and orbit-orbit term in form

$$
H_{R S}=H_{M C}+H_{D 1}+H_{D 2}+H_{S S C}+H_{O O}
$$

where

$$
\begin{aligned}
& H_{M C}=-\frac{\alpha^{2}}{8} \sum_{i=1}^{N}\left(\nabla_{i}^{2}\right)^{\dagger} \nabla_{i}^{2} \\
& H_{D 1}=-\frac{\alpha^{2} Z}{8} \sum_{i=1}^{N}\left(\nabla_{i}^{2}\right)\left(\frac{1}{r_{i}}\right) \\
& H_{D 2}=\frac{\alpha^{2}}{4} \sum_{i<j}^{N}\left(\nabla_{i}^{2}\right)\left(\frac{1}{r_{i j}}\right)
\end{aligned}
$$




$$
\begin{aligned}
& H_{S S C}=-\frac{8 \pi \alpha^{2}}{3} \sum_{i<j}^{N}\left(s_{i} \cdot s_{j}\right) \delta\left(r_{i} \cdot r_{j}\right) \\
& H_{O O}=-\frac{\alpha^{2}}{2} \sum_{i<j}^{N}\left[\frac{p_{i} \cdot p_{j}}{r_{i j}}+\frac{r_{i j}\left(r_{i j} \cdot p_{i}\right) p_{j}}{r_{i j}^{3}}\right] .
\end{aligned}
$$

Fine-structure Hamiltonian $H_{F S}$ consists of the spin-orbit, spin-other-orbit, and spin-spin terms,

$$
H_{F S}=H_{S O}+H_{S O O}+H_{S S}
$$

where

$$
\begin{aligned}
& H_{S O}=\frac{\alpha^{2} Z}{2} \sum_{i=1}^{N}\left(\frac{1}{r_{i}^{3}}\right) l_{i} \cdot s_{i} \\
& H_{S O O}=-\frac{\alpha^{2}}{2} \sum_{i<j}^{N} \frac{r_{i j} \times p_{i}}{r_{i j}^{3}}\left(s_{i}+2 s_{j}\right) \\
& H_{S S}=\alpha^{2} \sum_{i<j}^{N} \frac{1}{r_{i j}^{3}}\left[s_{i} \cdot s_{j}-3 \frac{\left(s_{i} \cdot r_{i j}\right)\left(s_{j} \cdot r_{i j}\right)}{r_{i j}^{2}}\right] .
\end{aligned}
$$

The Breit-Pauli wave functions are obtained as a linear combination of the form

$$
\Psi(\gamma J M)=\sum_{i=1}^{M} c_{i} \Phi\left(\gamma_{i} L_{i} S_{i} J M\right)
$$

where $\Phi(\gamma L S J M)$ are $L S J$ coupled configuration state functions (CSFs), that is,

$$
\Phi(\gamma L S J M)=\sum_{M_{L} M_{S}}\left\langle L M_{L} S M_{S} \mid L S J M\right\rangle \Phi\left(\gamma L M_{L} S M_{S}\right),
$$

and $\gamma_{i}$ denotes configurations; the orbital $L_{i}$ and the spin $S_{i}$ angular momenta are coupled to give the total angular momentum $J$. The mixing (or expansion) coefficients $c_{i}$ are obtained by diagonalizing the Breit-Pauli Hamiltonian. The radial functions building the CSFs are taken from a previous non-relativistic MCHF calculation and only the expansion coefficients are optimized. The matrix eigenvalue problem becomes

$$
H c=E c,
$$


where $\boldsymbol{H}$ is the Hamiltonian matrix with elements

$$
H_{i j}=\left\langle\gamma_{i} L_{i} S_{i} J M\left|H_{B P}\right| \gamma_{j} L_{j} S_{j} J M\right\rangle
$$

and $\boldsymbol{c}=\left(c_{1}, \ldots, c_{M}\right)^{t}$ is the column vector of the expansion coefficients. The Breit-Pauli Hamiltonian is a first-order perturbation correction to the nonrelativistic Hamiltonian.

\section{$3 \quad$ Results and Discussion}

We have presented the calculation values of electron affinities for arsenic, antimony and bismuth, and the fine-structure level energy differences of the ground state of the negative ions (anion) of these atoms. The calculations have been performed using the MCHF atomic structure code [6] based on the multiconfiguration Hartree-Fock method [5] within the framework of the BreitPauli Hamiltonian for relativistic effects in addition to correlation effects.

Table 1 Configurations selected in calculations.

\begin{tabular}{cl}
\hline Atoms/anions & \multicolumn{1}{c}{ Configurations } \\
\hline & $4 s^{2} 4 p^{3}+4 s^{2} 4 p^{2} 5 p+4 s^{2} 4 p 4 d^{2}+4 s^{2} 4 p 4 f^{2}$ \\
& $+4 s 4 p^{3} 4 d+4 s 4 p^{3} 5 d+4 s 4 p^{3} 5 s+4 s 4 p^{2} 4 d 4 f+4 p^{3} 4 f^{2}$ \\
\hline & $4 s^{2} 4 p^{4}+4 s^{2} 4 p^{3} 5 p+4 s^{2} 4 p^{3} 4 f+4 s^{2} 4 p^{3} 5 f$ \\
& $+4 s^{2} 4 p^{2} 4 d^{2}+4 s^{-} 4 p^{4} 4 d+4 s 4 p^{4} 5 s+4 s 4 p^{4} 5 d$ \\
& $+4 p^{4} 5 d^{2}+4 s 4 p^{3} 4 d 4 f+4 p^{4} 4 f^{2}$ \\
\hline & $5 s^{2} 5 p^{3}+5 s^{2} 5 p^{2} 6 p+5 s^{2} 5 p 5 d^{2}+5 s^{2} 5 p 4 f^{2}+5 s 5 p^{3} 5 d+$ \\
& $5 s 5 p^{3} 6 d+5 s 5 p^{3} 6 s+5 p^{3} 5 d^{2}+5 s 5 p^{2} 5 d 5 f+5 p^{3} 4 f^{2}$ \\
& $5 s^{2} 5 p^{4}+5 s^{2} 5 p^{3} 6 p+5 s^{2} 5 p^{3} 4 f+5 s^{2} 5 p^{3} 5 f$ \\
& $+5 s^{2} 5 p^{3} 6 f+5 s^{2} 5 p^{2} 5 d^{2}+5 s 5 p^{4} 5 d+5 s 5 p^{4} 6 s$ \\
& $+5 p^{4} 5 d^{2}+5 s 5 p^{3} 5 d 5 f+5 p^{4} 4 f^{2}$ \\
\hline & $6 s^{2} 6 p^{3}+6 s^{2} 6 p^{2} 7 p+6 s^{2} 6 p 6 d^{2}+6 s^{2} 6 p 5 f^{2}+6 s 6 p^{3} 6 d$ \\
& $+6 s 6 p^{3} 7 d+6 s 6 p^{3} 7 s+6 p^{3} 6 d^{2}+6 s 6 p^{2} 6 d 6 f+6 p^{3} 5 f^{2}$ \\
& $6 s^{2} 6 p^{4}+6 s^{2} 6 p^{3} 7 p+6 s^{2} 6 p^{3} 5 f+6 s^{2} 6 p^{3} 6 f$ \\
& $+6 s^{2} 6 p^{3} 7 f+6 s^{2} 6 p^{2} 6 d^{2}+6 s 6 p^{4} 6 d+6 s 6 p^{4} 7 d$ \\
& $+6 s 6 p^{4} 7 s+6 p^{4} 6 d^{2}+6 s 6 p^{3} 6 d 6 f+6 p^{4} 5 f^{2}$ \\
\hline
\end{tabular}

We have obtained the electron affinity values of $0.2702 \mathrm{eV}, 0.6054 \mathrm{eV}$ and $0.1466 \mathrm{eV}$ for arsenic, antimony and bismuth, respectively. These values include only correlation effects. When the relativistic effects in addition to 
correlation effects were considered, the values of $0.3278 \mathrm{eV}, 0.7305 \mathrm{eV}$ and $0.5649 \mathrm{eV}$ were found for these anions, respectively. The values are in agreement with those in [13] for arsenic, [14] for antimony and [15] for bismuth. The electron affinity for bismuth is somewhat poor. Probably the hyperfine-structure levels for this ion have to be investigated. This work is restricted only to the fine-structure levels for the ground states of the anions mentioned above.

Table 1 displays the configurations considered in our calculations. We have also used various other configuration sets, as given in Table 1. Due to computer constraints and code limits, we did not use any more configuration sets. The calculations for antimony and bismuth have been performed using the configuration sets given in [8]. These atoms and their anions have many more electrons. It is well known that these atoms and anions have half-filled $\mathrm{p}$ shells in the ground state valence configuration. These configurations produce many more configuration state functions. Therefore we have restricted our calculations to the ground state configuration state functions only.

Table 2 The energy differences, $\Delta \mathrm{E}$ (in $\mathrm{eV}$ ), between fine-structure levels for ground state of $\mathrm{As}^{-}, \mathrm{Sb}^{-}$, and $\mathrm{Bi}^{-}$.

\begin{tabular}{|c|c|c|c|c|c|c|}
\hline \multirow{2}{*}{ Term } & \multicolumn{2}{|c|}{$\mathbf{A s}^{-}$} & \multicolumn{2}{|c|}{$\mathbf{S b}^{-}$} & \multicolumn{2}{|c|}{$\mathbf{B i}^{-}$} \\
\hline & $\boldsymbol{\Delta} \boldsymbol{E}_{\text {This work }}$ & $\boldsymbol{\Delta} \boldsymbol{E}_{\text {Other works }}$ & $\boldsymbol{\Delta} \boldsymbol{E}_{\text {This work }}$ & $\boldsymbol{\Delta} \boldsymbol{E}_{\text {Other works }}$ & $\boldsymbol{\Delta} \boldsymbol{E}_{\text {This work }}$ & $\Delta \boldsymbol{E}_{\text {Other works }}$ \\
\hline \multirow{2}{*}{${ }^{3} \mathrm{P}_{0}-{ }^{3} \mathrm{P}_{1}$} & 0.0544 & - & 0.1157 & $0.0144^{\mathrm{e}}$ & 0.3123 & - \\
\hline & $0.0473 *$ & - & & & & \\
\hline \multirow{3}{*}{${ }^{3} \mathrm{P}_{1}-{ }^{3} \mathrm{P}_{2}$} & 0.1135 & $0.125^{\mathrm{a}}$ & 0.2457 & $0.33268^{\mathrm{e}}$ & 0.7877 & - \\
\hline & $0.1301 *$ & $0.1276^{\mathrm{c}}$ & & $0.33957^{\mathrm{b}}$ & & \\
\hline & & $0.127^{\mathrm{d}}$ & & & & \\
\hline \multirow{4}{*}{${ }^{3} \mathrm{P}_{0}-{ }^{3} \mathrm{P}_{2}$} & 0.1678 & $0.166^{\mathrm{a}}$ & 0.3615 & $0.3471^{\mathrm{e}}$ & 1.1000 & - \\
\hline & $0.1775^{*}$ & $0.17^{\mathrm{b}}$ & & & & \\
\hline & & $0.1643^{c}$ & & & & \\
\hline & & $0.161^{\mathrm{d}}$ & & & & \\
\hline
\end{tabular}

${ }^{\mathrm{a}}$ Haeffler, et al. [3]; ${ }^{\mathrm{b}}$ Feldmann, et al. [11]; ${ }^{\mathrm{c}}$ Walter, et al. [13]; ${ }^{\mathrm{d}}$ Lippa, et al. [12]; ${ }^{\mathrm{e}}$ Scheeret, et al. [14].

The energy differences, $\Delta \mathrm{E}$ (in $\mathrm{eV}$ ), between the fine-structure levels for the ground state of $\mathrm{As}^{-}, \mathrm{Sb}^{-}$and $\mathrm{Bi}^{-}$are given in Table 2 . In this table the values denoted by the superscript $*$ for $\mathrm{As}^{-}$have been obtained using the configuration 
set $4 s^{2} 4 p^{4}, 4 s^{2} 4 p^{3} 5 p, 4 s^{2} 4 p^{3} 4 f, 4 s^{2} 4 p^{3} 5 f, 4 s^{2} 4 p^{3} 6 f, 4 s^{2} 4 p^{2} 4 d^{2}, 4 s^{2} 4 p^{2} 5 d^{2}, 4 s 4 p^{4} 4 d$, $4 s 4 p^{4} 5 s, 4 s 4 p^{4} 5 d, 4 p^{6}, 4 p^{4} 5 d^{2}, 4 s 4 p^{3} 4 d 4 f, 4 p^{4} 4 f^{2}, 4 p^{4} 5 f^{2}$. An agreement is seen for $\mathrm{As}^{-}$and $\mathrm{Sb}^{-}$when our results were compared with other works. We have made the same calculations for nitrogen and phosphorus anions [7]. The energy differences between the fine-structure levels for phosphorus anions are in agreement with other works. This agreement can be also seen for As and $\mathrm{Sb}$ anions, as shown in Table 2. Therefore we think that the results obtained for bismuth anions are correct. As we know, there are no data on the fine structure of the negative ion of bismuth in the literature.

\section{Conclusion}

The aim of this studywas to investigate the correlation and relativistic effects on the behavior of electron affinities of arsenic, antimony and bismuth using the MCHF method. Also, we have obtained the ground state fine-structure levels of the anions of these atoms. It is noted in [17] that the correlation and relativistic effects must be included in calculations, especially for negative ions. In addition, electron affinity has been regarded as one of the hardest atomic or molecular properties to reproduce in an ab initio quantum mechanical calculation. The electron affinity of an atom is a measure of the stability of the corresponding negative ion.

\section{Acknowledgments}

The authors are very grateful to the anonymous reviewers and editors for their stimulating comments and valuable suggestions, which resulted in improvements to this paper.

\section{References}

[1] Buckman, S.J. \& Clark, C.W., Atomic Negative-Ion Resonances, Rev. Mod. Phys., 66(2), pp. 539-655, 1994.

[2] Blondel, C., Recent Experimental Achievements with Negative Ions, Phys. Scr., T58, pp. 31-42, 1995.

[3] Haeffler, G., Ljungblad, U., Kiyan, I.Y. \& Hanstorp, D., Fine Structure of $A s^{-}$, Z. Phys. D: At., Mol. Clusters, 42, pp. 263-266, 1997.

[4] Koga, T., Aoki, H., Garcia de la Vega, J.M. \& Tatewaki, H., Atomic Ionization Potentials and Electron Affinities with Relativistic and Mass Corrections, Theor. Chem. Acc., 96, pp. 248-255, 1997.

[5] Fischer, C.F., Brage, T. \& Jönsson, P., Computational Atomic Structure, an MCHF Approach, Institute of Physics Publishing, Bristol and Philadelphia, England and USA, 1997. 
[6] Fischer, C.F., The MCHF Atomic-Structure Package, Computer Physics Communications, 128, pp. 635-636, 2000.

[7] Özdemir, L., Atik, N. \& Konan, G.G., The Fine Structure Levels for Ground States of Negative Ions of Nitrogen and Phosphorus, SAU Fen Bilimleri Enstitüsü Dergisi- SAU Journal of Science, 17, pp. 139-146, 2013.

[8] Biémont, E. \& Quinet, P., Forbidden Lines in $6 p^{k}(k=1-5)$ Configurations, Physica Scripta, 54, pp. 36-43, 1996.

[9] Kramida, A.E., Atomic Energy Levels and Spectra Bibliographic Database (version 2.0), http://physics.nist.gov/Elevbib, National Institute of Standards and Technology, Gaithersburg, MD., October 5, 2010, (April 16, 2013).

[10] Andersen, T., Atomic Negative Ions: Structure, Dynamics And Collisions, Phys. Reports, 394, pp.157-313, 2004.

[11] Feldmann, D., Rackwitz, R., Heinicke, E. \& Kaiser, H.J., Photodetachment of Some Atomic Negative Ions: $\mathrm{P}^{-}, \mathrm{As}^{-}, \mathrm{Sb}^{-}, \mathrm{Bi}^{-}, \mathrm{Te}^{-}$, $\mathrm{Cr}^{-}, \mathrm{Ni}^{-}$, Z. Phys. A, 282, pp. 143-148, 1977.

[12] Lippa, T.P., Xu, S., Lyapustina, S.A., Nilles, J.M. \& Bowen, K.H., Photoelectron Spectroscopy of $\mathrm{As}^{-}, \mathrm{As}_{2}{ }^{-}, \mathrm{As}_{3}{ }^{-}, \mathrm{As}_{4}{ }^{-}$, and $A s_{5}{ }^{-}$, J. Chem. Phys., 109, pp. 10727-10731, 1998.

[13] Walter C.W., Gibson, N.D., Field III, R.L., Snedden, A.P., Shapiro, J.Z., Janczak, C.M. \& Hanstorp, D., Electron Affinity of Arsenic and The Fine Structure of $A s^{-}$Measured Using Infrared Photodetachment Threshold Spectroscopy, Phys. Rev. A, 80, pp. 014501 (4 pages), 2009.

[14] Scheer, M., Haugen, H.K. \& Beck, D.R., Single and Multiphoton Infrared Laser Spectroscopy of $\mathrm{Sb}^{-}$: A Case Study, Phys. Rev. Lett., 79, pp. 4104-4107, 1997.

[15] Feigerle, C.S., Corderman, R.R. \& Lineberger, W.C., Electron Affinities of $B, A l, B i$, and $P b$, J. Chem. Phys., 74, pp. 1513 (3 pages), 1981.

[16] Bilodeau, R.C. \& Haugen, H.K., Electron Affinity of Bi Using Infrared Laser Photodetachment Threshold Spectroscopy, Phys. Rev. A, 64, pp.024501-024503, 2001.

[17] Andersson, P., Lindahl, A.O., Alfredsson, C., Rogström, L., Diehl, C., Pegg, D.J. \& Hanstorp, D., The Electron Affinity of Phosphorus, J. Phys. B: At. Mol. Opt. Phys., 40, pp.4097-4107, 2007. 